Discrete Comput Geom 29:375-393 (2003)

DOI: $10.1007 / \mathrm{s} 00454-002-2840-2$

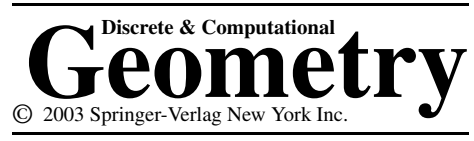

\title{
On Levels in Arrangements of Curves*
}

\author{
Timothy M. Chan \\ Department of Computer Science, University of Waterloo, \\ Waterloo, Ontario N2L 3G1, Canada \\ tmchan@uwaterloo.ca
}

\begin{abstract}
Analyzing the worst-case complexity of the $k$-level in a planar arrangement of $n$ curves is a fundamental problem in combinatorial geometry. We give the first subquadratic upper bound (roughly $O\left(n k^{1-1 /\left(9 \cdot 2^{s-3}\right)}\right)$ ) for curves that are graphs of polynomial functions of an arbitrary fixed degree $s$. Previously, nontrivial results were known only for the case $s=1$ and $s=2$. We also improve the earlier bound for pseudo-parabolas (curves that pairwise intersect at most twice) to $O\left(n k^{7 / 9} \log ^{2 / 3} k\right)$. The proofs are simple and rely on a theorem of Tamaki and Tokuyama on cutting pseudo-parabolas into pseudo-segments, as well as a new observation for cutting pseudo-segments into pieces that can be extended to pseudo-lines. We mention applications to parametric and kinetic minimum spanning trees.
\end{abstract}

\section{Introduction}

Suppose we have $n$ elements on the real line, each moving continuously according to some polynomial function of degree $s$. How many times can the $k$ th smallest element change over time?

We can state this natural question more precisely in geometric terms by viewing the $x$-coordinate as time: given an arrangement of $n$ curves in the plane formed by graphs of degree-s polynomial functions, how many vertices can the $k$-level (see Fig. 1)

$$
\left\{q \in \mathbb{R}^{2} \mid q \text { is on exactly one curve and above exactly } k-1 \text { other curves }\right\}
$$

have in the worst case? More generally, we can ask the same question for an arbitrary family of s-intersecting curves (or curve segments), where each curve (curve segment) is continuous, intersects each vertical line once (at most once), and intersects another curve (curve segment) at most $s$ times.

\footnotetext{
* A preliminary version of this paper appeared in Proceedings of the 41st IEEE Symposium on the Foundations of Computer Science [18]. This research was supported by an NSERC Research Grant.
} 


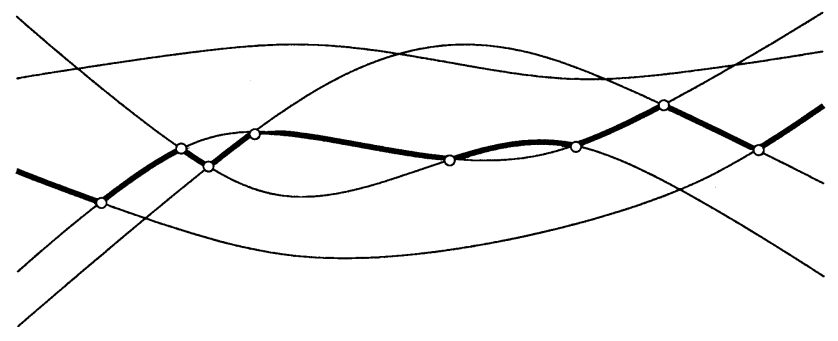

Fig. 1. The 3-level in an arrangement of five 3-intersecting curves.

The motivation comes from several directions. First, our problem is among the simplest in the combinatorial analysis of so-called "kinetic data structures," an area that has received much recent attention, dealing with the maintenance of structures of objects in motion [11], [31]; in particular, there is a direct relevance to the study of kinetic and parametric minimum spanning trees [26], [32], [36] (see below). Generally, arrangements of curves and surfaces have for a long time been a central topic in computational geometry [6], [23], [24], [33], [45], and levels in arrangements have been used in the design of algorithms for range searching [4], [19], geometric optimization with $k$ violations [28], [39], and partitioning of point sets [15], [38] (see the surveys [6], [8], and [33] and the books [23], [40], [41], and [45] for more details).

History. The $k$-level has a reputation of being one of the more difficult substructures of arrangements to analyze, even in the simplest case of lines $(s=1)$ in the plane, where in the dual, it is known more famously under the name of the $k$-set problem (given an $n$-point set $P$ in the plane, bound the number of subsets of size $k$ that can be formed by intersecting $P$ with a halfplane). In the early 1970s, Erdôs et al. [27] and Lovász [37] started the investigation by establishing a nontrivial $O(n \sqrt{k})$ upper bound and an $\Omega(n \log k)$ lower bound, but an improvement did not come until 1989, when Pach et al. [42] managed to reduce the upper bound by a small $\log ^{*} k$ factor. In 1997 a breakthrough $O\left(n k^{1 / 3}\right)$ upper bound was obtained by Dey [21] with a short elegant proof; Dey's result remains the current record.

Like the classical proof, Dey's proof generalizes to any arrangement of 1-intersecting curves, commonly called pseudo-lines [23], [30], as shown by Tamaki and Tokuyama [47]. Both proofs can also be adapted for an arrangement of line segments, the latter yielding an $O\left(n k^{1 / 3} \alpha(n / k)\right)$ bound, as shown by Agarwal et al. [1], where $\alpha(\cdot)$ denotes the inverse Ackermann function. (Note that the worst-case complexity of the 1-level, i.e., lower envelope, of $n$ line segments is $\Theta(n \alpha(n))$ [45].) Whether the same bound holds for an arbitrary arrangement of pseudo-segments (1-intersecting curve segments) appears to be open, because, as noted by Agarwal et al., the known techniques apply only to a special class of pseudo-segments which we call extendible pseudo-segments (see Section 2).

Analyzing the $k$-level complexity for more general families of curves appears even more challenging, as the preceding techniques simply fail. The first nontrivial result for quadratic functions $(s=2)$ was obtained only in 1995 by Tamaki and Tokuyama [48]. They proved a theorem on how to $c u t$ an arrangement of pseudo-parabolas (2-intersecting 
curves) into an arrangement of pseudo-segments. When combined with the classical result for pseudo-lines, this theorem allows them to derive a subquadratic $O\left(n^{23 / 12}\right)$ bound on the $k$-level of pseudo-parabolas. With Dey's improvement and the " $k$-sensitizing" techniques by Agarwal et al. [1], the bound can be directly reduced to $O\left(n k^{8 / 9}\right)$.

Unfortunately, one cannot derive nontrivial $k$-level bounds for general $s$-intersecting curves for $s=3$ or higher with this particular approach, because, as Tamaki and Tokuyama [48] observed, the worst-case bound for cutting an arrangement of 3-intersecting curves into a pseudo-segment arrangement is quadratic. Perhaps because of this, there has been no successful attempt since on further generalization (despite the need in some of the above-mentioned applications).

Regarding worst-case lower bounds for the $k$-level of $s$-intersecting curves, no better results are known other than Klawe, Paterson, and Pippenger's bound of $n 2^{\Omega(\sqrt{\log k})}$ for pseudo-lines $(s=1)$ from the 1980s [23]. Toth [49] recently proved a lower bound of the same form for lines.

New Results. The first result of this paper is an $O\left(n k^{1 / 3} \alpha(n / k) \log ^{2 / 3} k\right)$ bound for the $k$-level of an arbitrary arrangement of $n$ pseudo-segments. Our approach is to cut such an arrangement into an arrangement of extendible pseudo-segments, for which we can apply the known bound; the method of cutting is quite simple and is based on a segment tree. Along the way, we describe a simple characterization, of independent interest, for when a family of pseudo-segments is extendible.

Our new-found observations on pseudo-segments also allow us to improve Tamaki and Tokuyama's bound for the $k$-level of pseudo-parabolas further to $O\left(n k^{7 / 9} \log ^{2 / 3} k\right)$.

Our main contribution, though, is the first subquadratic combinatorial result on the $k$-level of degree-s polynomial functions for any fixed constant $s$. The initial version of our proof yields an $O\left(\lambda_{s}(n / k) k^{2-2 / 3^{s}} \log ^{1-1 / 3^{s-1}} k\right)$ bound [18]; an improved version of the proof yields $O\left(\lambda_{s}(n / k) k^{2-1 /\left(9 \cdot 2^{s-3}\right)} \log ^{2 / 3} k\right)$. Here, $\lambda_{s}(\cdot)$ is an almost linear bound on the complexity of the lower envelope [45]. The approach is also by cutting the arrangement, but despite the aforementioned problem with this approach, we circumvent it curiously by exploiting the analytical properties of the curves rather than the combinatorial properties alone-a sharp departure from earlier proofs ( $s=1$ or 2) where all results for degree-s polynomials (linear or quadratic functions) adapt to arbitrary $s$-intersecting curves (pseudo-lines or pseudo-parabolas). Our basic idea is surprisingly simple: cut the arrangement of the derivatives of the functions recursively; at each step, we apply Tamaki and Tokuyama's cut theorem for pseudo-parabolas, together with random sampling.

Despite the weak exponent $2-1 /\left(9 \cdot 2^{s-3}\right)$ in our $k$-level bound, our work is important because it opens up many nontrivial combinatorial questions about arrangements of curves in the plane that previously we were not ready to raise. Traditionally, most studies in arrangements of curves assume only an $s$-intersecting condition, but our work indicates interestingly that polynomial curves and general $s$-intersecting curves can have quite different combinatorial characteristics.

Applications. Our approach gives new results in a number of related problems as well. For instance, we obtain new bounds for the complexity of $k$ nonoverlapping pseudoconcave chains in an arrangement of curves. By known reductions [26], [32], this leads 
to a subquadratic upper bound (in the number of edges) on the number of changes to a parametric minimum spanning tree (or, more generally, a parametric matroid optimization) if the edge weights vary polynomially in time. We also derive the first subcubic bound on the number of changes to a Euclidean minimum spanning tree of $n$ linearly moving points in any fixed dimension [36]. Very recently, the techniques of this paper have also found applications in other fundamental problems of combinatorial geometry, concerning point-curve incidences [10] and the complexity of many faces in curve arrangements [2].

Other Related Work. The algorithmic problem of constructing the $k$-level of lines and curves has been examined by many researchers; for recent work, see [17] and [34]. The $k$-level problem in three dimensions has also received considerable attention in the linear case (arrangements of planes or triangles); see [1], [9], [12], [22], [25], [35], and [46]. A nontrivial combinatorial bound for hyperplanes in higher dimensions was known [50]. However, there has so far been no successful generalization to families of nonlinear surfaces.

In contrast, tight worst-case bounds on the 1-level, 2-level, $\ldots, k$-level combined are easier to obtain. An $O(n k)$ bound for lines was well known [7], [29]. Clarkson and Shor [20] gave a proof by random sampling that extends to higher dimensions. As shown by Sharir [44], this proof also yields an $O(n k \alpha(n / k))$ bound for pseudo-segments, and an $O\left(\lambda_{s}(n / k) k^{2}\right)$ bound for general $s$-intersecting curves.

\section{Extendible Pseudo-Segments}

Agarwal et al. [1] observed that the classical proof for the $k$-level of lines can be modified to yield an $O\left(n^{3 / 2}\right)$ bound for the $k$-level of $n$ line segments. They remarked that the same result can be obtained for a family of pseudo-segments (1-intersecting curve segments), provided that the following additional criterion holds: we can find a curve (the "extension") through each of the given curve segments such that the resulting family of curves is a pseudo-line family. We call such a family of pseudo-segments extendible. Not every family of pseudo-segments is extendible; see Fig. 2(a) for a minimal counterexample.

With the discovery of Dey's improved bound for $k$-levels and a certain lemma of Tamaki and Tokuyama [48] on pseudo-lines, we now have an $O\left(n^{4 / 3}\right)$ bound for the $k$-level of any arrangement of $n$ extendible pseudo-segments. This is the starting point

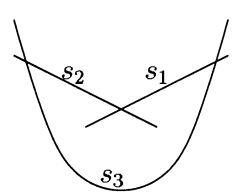

(a)

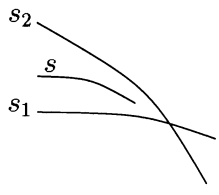

(b)

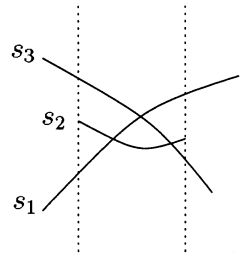

(c)

Fig. 2. (a) A nonextendible arrangement of pseudo-segments. (b) Proof of Lemma 3.1. (c) Proof of Lemma 3.2. 
for all our $k$-level results. However, for forthcoming applications, we need to make this bound sensitive to the number of intersections, as stated in the theorem below. We give a simple proof inspired by one of Tamaki and Tokuyama [48], using divide-and-conquer by slabs. (Alternatively, we can use divide-and-conquer by random sampling; for example, see the proofs of Theorems 5.1 and 8.1.)

Theorem 2.1. The $k$-level in an arrangement of $N$ extendible pseudo-segments with $X$ intersecting pairs has $O\left(N+N^{2 / 3} X^{1 / 3}\right)$ vertices.

Proof. Partition the plane into $\lceil N / e\rceil$ vertical slabs so that each slab has at most $e$ endpoints. Let $X_{i}$ be the number of intersections at slab $i$. Let $T_{i}$ be the subset of pseudosegments that have an endpoint inside slab $i$. Let $S_{i}$ be the subset of pseudo-segments not in $T_{i}$ that contribute to the $k$-level inside the slab.

Clearly, $T_{i}=O(e)$. We also have $\left|S_{i}\right|=O\left(\sqrt{X_{i}}+e\right)$ by the following argument: a segment in $S_{i}$ has at least $|j-k|-e$ intersections in slab $i$ if it is the $j$ th lowest at the left wall of the slab; consequently, at least $\left|S_{i}\right|-2 A$ segments have at least $A-e$ intersections; setting $A=\left|S_{i}\right| / 4$ implies that $X_{i}=\Omega\left(\left(\left|S_{i}\right| / 4-e\right)^{2}\right)$.

Since the $k$-level in the original arrangement coincides with a level of $S_{i} \cup T_{i}$ when restricted to slab $i$, the known result for extendible pseudo-segments gives the following upper bound on the entire $k$-level (by Hölder's inequality):

$$
O\left(\sum_{i=1}^{\lceil N / e\rceil}\left|S_{i} \cup T_{i}\right|^{4 / 3}\right)=O\left(\sum_{i=1}^{\lceil N / e\rceil}\left(X_{i}^{2 / 3}+e^{4 / 3}\right)\right)=O\left((N / e)^{1 / 3} X^{2 / 3}+N e^{1 / 3}\right) .
$$

The theorem follows by setting $e=\lceil X / N\rceil$.

Remark. The original proof by Tamaki and Tokuyama [48] implies a weaker bound of $O\left(N+N^{1 / 3} X^{2 / 3}\right)$ but does not require extendibility. It uses the known result for pseudo-lines rather than pseudo-segments, but this requires partitioning the plane by vertical lines at every endpoint (in other words, we have to set $e=1$ ).

Tamaki and Tokuyama's proof thus implicitly gives an $O\left(n^{5 / 3}\right)$ bound for the $k$-level in an arrangement of $n$ arbitrary pseudo-segments (plug in $N=n$ and $X=O\left(n^{2}\right)$ ). We give a better result in the next section.

\section{Pseudo-Segments}

To obtain a good bound on the $k$-level of general pseudo-segments, we cut the arrangement in such a way that the result from the previous section can be applied. Cutting the arrangement (an idea from Tamaki and Tokuyama's paper [48]) simply means selecting a set of cut points and breaking each segment into subsegments by removing an infinitesimal neighborhood around each incident cut point. The $k$-level remains unchanged, except for the addition of infinitesimal edges.

We first characterize when a family $S$ of pseudo-segments is extendible. Define a directed graph $\mathcal{G}(S)$ as follows: the vertices are pseudo-segments in $S$, and there is an 
edge between $s_{1}$ and $s_{2}$ whenever two pseudo-segments $s_{1}$ and $s_{2}$ intersect; the edge is oriented as $\left(s_{1}, s_{2}\right)$ if to the left of the intersection, $s_{1}$ is below $s_{2}$. (For example, in Fig. 2(a) the edge set of $\mathcal{G}(S)$ is $\left\{\left(s_{1}, s_{2}\right),\left(s_{2}, s_{3}\right),\left(s_{3}, s_{1}\right)\right\}$.)

Lemma 3.1. A family $S$ of pseudo-segments is extendible if and only if $\mathcal{G}(S)$ is acyclic.

Proof. The "only if" part is obvious, because for each edge $\left(s_{1}, s_{2}\right) \in \mathcal{G}(S)$, the extension of $s_{1}$ is below the extension of $s_{2}$ at $x=-\infty$.

To prove the "if" part, let $\prec$ be a topological order of $\mathcal{G}(S)$ (i.e., a total order on $S$ such that $\left(s_{1}, s_{2}\right) \in \mathcal{G}(S)$ implies $\left.s_{1} \prec s_{2}\right)$. Take any segment $s \in S$. We will extend it at either endpoint while maintaining the pseudo-segment property and ensuring that $\prec$ remains a topological order of $\mathcal{G}(S)$.

Certainly, we can extend $s$ to the right as long as there is no new crossing. A problem occurs only when the right endpoint approaches the intersection of a pair of segments $s_{1}$ and $s_{2}$, as shown in Fig. 2(b), with the right endpoint of $s$ above $s_{1}$ and below $s_{2}$. Then $s_{1} \prec s_{2}$, so one or both of the following two cases must hold:

Case 1: $s_{1} \prec s$. Then $s_{1}$ and $s$ cannot intersect. So we can extend $s$ to cross $s_{1}$, and $S$ is still a pseudo-segment family. This causes the addition of the edge $\left(s_{1}, s\right)$ to $\mathcal{G}(S)$, but $\prec$ is still a topological order.

Case 2: $s \prec s_{2}$. Then $s$ and $s_{2}$ cannot intersect. We extend $s$ to cross $s_{2}$ instead. The invariants again are maintained, this time with the addition of the edge $\left(s, s_{2}\right)$ to $\mathcal{G}(S)$.

Now we can continue the process until the right endpoint approaches $x=\infty$ (the process is finite since each iteration adds a new intersection). Similarly, we can extend the left endpoint to $x=-\infty$. Repeating the process for each of the segments in turn results in a family of pseudo-lines.

We can now give a simple sufficient condition for when pseudo-segments are extendible. Some terminology first: the $x$-interval of a pseudo-segment refers to the range of its $x$-values; two intervals cross if their interiors intersect and one is not contained in the other.

Lemma 3.2. If $S$ is a family of pseudo-segments such that no two $x$-intervals cross, then $\mathcal{G}(S)$ is acyclic.

Proof. Suppose $\mathcal{G}(S)$ has a cycle. Let $\left\langle s_{1}, \ldots, s_{\ell}, s_{1}\right\rangle$ be a shortest cycle then $(\ell \geq 3)$. Without loss of generality, say $s_{2}$ has the shortest $x$-interval. Then the $x$-intervals of $s_{1}$ and $s_{3}$ must contain the $x$-interval of $s_{2}$, so the left endpoint of $s_{2}$ is above $s_{1}$ but below $s_{3}$, and the right endpoint of $s_{2}$ is below $s_{1}$ but above $s_{3}$ (for example, see Fig. 2(c)). However, this implies that $\left(s_{1}, s_{3}\right) \in \mathcal{G}(S)$, so $\left\langle s_{1}, s_{3}, \ldots, s_{\ell}, s_{1}\right\rangle$ would be a shorter cycle: contradiction.

Our key result regarding pseudo-segments can now be proved by a standard segmenttree idea [43]. 
Theorem 3.3. Any arrangement of $n$ pseudo-segments can be cut into an arrangement of $O(n \log n)$ extendible pseudo-segments.

Proof. Given $n$ intervals, the standard tree construction gives us a collection of canonical subintervals, no two of which cross, such that each interval can be partitioned into $O(\log n)$ canonical subintervals. Apply this observation to the $x$-intervals of the pseudo-segments, break the pseudo-segments at the corresponding $x$-values, and invoke Lemmas 3.2 and 3.1 .

Now, a $k$-level bound of $O\left(n^{4 / 3} \log ^{2 / 3} n\right)$ can be obtained by applying Theorem 2.1 to the output of Theorem 3.3, with $N=O(n \log n)$ and $X=O\left(n^{2}\right)$. Agarwal et al. [1] described general techniques for making $k$-level bounds sensitive to $k$. In this instance, we then have:

Corollary 3.4. The k-level in an arrangement of $n$ pseudo-segments has $O\left(n k^{1 / 3} \alpha(n / k) \log ^{2 / 3} k\right)$ vertices.

\section{Pseudo-Parabolas}

Tamaki and Tokuyama [48] obtained a nontrivial bound for the $k$-level of pseudoparabolas (2-intersecting curves) by proving the following key theorem: any arrangement of $n$ pseudo-parabolas can be cut into an arrangement of $O\left(n^{5 / 3}\right)$ pseudo-segments. (See Section 7 on how.) Their method, combined with Dey's pseudo-line bound, then yields an $O\left(n^{17 / 9}\right)$ bound for the $k$-level (by the Remark after Theorem 2.1, with $N=O\left(n^{5 / 3}\right)$ and $\left.X=O\left(n^{2}\right)\right)$.

By combining Tamaki and Tokuyama's theorem with Theorem 3.3 instead, any arrangement of $n$ pseudo-parabolas can be cut into an arrangement of $O\left(n^{5 / 3} \log n\right)$ extendible pseudo-segments. Consequently, we can apply Theorem 2.1 with $N=O$ $\left(n^{5 / 3} \log n\right)$ and $X=O\left(n^{2}\right)$ to get an improved $O\left(n^{16 / 9} \log ^{2 / 3} n\right)$ bound for the $k$-level. When made $k$-sensitive with the techniques of Agarwal et al. [1] mentioned earlier, the bound becomes the following:

Corollary 4.1. The $k$-level in an arrangement of $n$ pseudo-parabolas has $O\left(n k^{7 / 9} \log ^{2 / 3} k\right)$ vertices.

\section{Extendible Pseudo-Parabolic Segments}

A family of extendible s-intersecting segments refers to a family of $s$-intersecting curve segments where we can find a curve through each of the given curve segments such that the resulting family of curves is $s$-intersecting. Trivially, Tamaki and Tokuyama's theorem applies to cutting an arrangement of extendible 2-intersecting (pseudo-parabolic) segments, by cutting their extensions, so roughly the same $k$-level bound as Corollary 4.1 holds. 
For the purpose explained in the next section, however, we need the following intersection-sensitive version of Tamaki and Tokuyama's cut theorem. We prove this by divide-and-conquer via a well-known random-sampling technique (specifically, the proper term for the required construction is an intersection-sensitive $(1 / r)$-cutting, as described in [14]).

Theorem 5.1. Any arrangement of $N$ extendible pseudo-parabolic segments with $X$ intersecting pairs can be cut into an arrangement of $O\left(N+N^{1 / 3} X^{2 / 3}\right)$ pseudo-segments, or $O\left(\left(N+N^{1 / 3} X^{2 / 3}\right) \log N\right)$ extendible pseudo-segments.

Proof. We employ a probabilistic method. Choose a random sample $R$ of $r$ segments and let $\operatorname{VD}(R)$ be the vertical decomposition of the arrangement of $R$ - a partitioning of the plane into trapezoids (for the precise definition, consult [40]). For each trapezoid $\Delta \in \mathrm{VD}(R)$, let $S_{\Delta}$ denote all the segments that intersect $\Delta$, clip these segments with $\Delta$, and apply Tamaki and Tokuyama's theorem to (the extensions of) $S_{\Delta}$ to break them into pseudo-segments. As a result, we obtain an arrangement of $O(M)$ pseudo-segments, where

$$
M=\sum_{\Delta \in \mathrm{VD}(R)}\left|S_{\Delta}\right|^{5 / 3}
$$

By Theorem 3.3, we can refine it into an arrangement of $O(M \log N)$ extendible pseudosegments.

Now, from the theory developed by Clarkson and Shor [20], [40], the expected value of $M$ is given by the expression $O\left(\eta(r) \cdot(N / r)^{5 / 3}\right)$, where $\eta(r)$ is the expected size of $\operatorname{VD}(R)$. Since the arrangement of $R$ has $O(r)$ endpoints and an expected number of $O\left(X(r / N)^{2}\right)$ intersections, we have $\eta(r)=O\left(r+X(r / N)^{2}\right)$. Hence,

$$
E[M]=O\left(\left(r+X(r / N)^{2}\right) \cdot(N / r)^{5 / 3}\right),
$$

and the theorem follows by setting $r=\left\lceil N^{2} / X\right\rceil$ (assuming $X \geq N$ ).

Discussion of general pseudo-parabolic segments can be found in Section 7.

\section{Polynomial Curves}

Logically, the next step is to consider arbitrary $s$-intersecting curves for $s \geq 3$, but generalization of the preceding techniques is hindered by the following disappointing observation made by Tamaki and Tokuyama [48]: although it is possible to cut any $s$ intersecting curves into subquadratically many $(s-1)$-intersecting curve segments for any even constant $s$, the same is not true for odd values of $s$. It is easy to construct, for example, a family of $n$ 3-intersecting curves that requires $\Omega\left(n^{2}\right)$ cuts: just take an arrangement of $n$ lines and modify the neighborhood of each intersection point so that we see three intersections instead.

However, if one attempts to draw the above worst-case example, one would discover that a large number of "oscillations" must occur in its curves-a suggestion that perhaps 


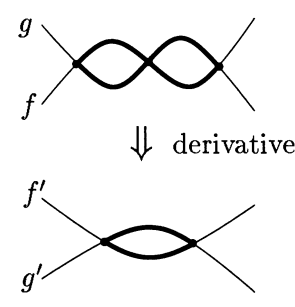

Fig. 3. If $f$ and $g$ intersect $s+1$ times, then $f^{\prime}$ and $g^{\prime}$ intersect at least $s$ times.

the example cannot be realized by low-degree polynomials like cubics. Indeed, this is the case. The following observation (with $s=2$ ) tells us, for instance, that we can cut cubic functions into pseudo-parabolic segments, simply by cutting their derivatives (which are quadratic!) into pseudo-segments by Tamaki and Tokuyama's theorem.

In what follows, we make no distinction between functions and their graphs. Thus, the derivative of a curve segment refers to the (graph of the) derivative of the underlying function. For a family $S$ of curve segments, $S^{\prime}$ denotes the family of the derivatives of the segments, and for a union $U$ of curve segments, $U^{\prime}$ denotes the union of the derivatives of the segments.

Observation 6.1. If $S^{\prime}$ forms a family of $(s-1)$-intersecting curve segments, then $S$ forms a family of s-intersecting curve segments. Furthermore, if the former family is extendible, so is the latter.

Proof. To prove the first part, suppose two curve segments $f, g \in S$ intersect $s+1$ or more times, say at $x$-coordinates $x_{1}, \ldots, x_{s+1}$ from left to right. Since $f\left(x_{i}\right)=g\left(x_{i}\right)$ and $f\left(x_{i+1}\right)=g\left(x_{i+1}\right)$, there exists some $a_{i} \in\left(x_{i}, x_{i+1}\right)$ such that $f^{\prime}\left(a_{i}\right)=g^{\prime}\left(a_{i}\right)$ by Rolle's theorem (i.e., the mean value theorem) for each $i=1, \ldots, s$. (See Fig. 3.) Thus, $f^{\prime}$ and $g^{\prime}$ intersect at least $s$ times: contradiction.

For the second part, say $f^{\prime}$ is extended to a total function denoted by $\widehat{f}^{\prime}$. We extend each function $f \in S$, defined on interval $[a, b]$, to the total function $\hat{f}(x):=f(a)+\int_{a}^{x} \widehat{f}^{\prime}(t) d t$ (which clearly agrees with $f$ on $[a, b])$. Since $\left\{(\hat{f})^{\prime}=\widehat{f}^{\prime} \mid f \in S\right\}$ is $(s-1)$-intersecting, the first part of the lemma implies that $\{\hat{f} \mid f \in S\}$ is $s$-intersecting.

By repeated applications of Observation 6.1 and Theorem 5.1, we obtain the first subquadratic bound for cutting degree-s polynomial curves into (extendible) pseudosegments:

Theorem 6.2. Any arrangement of $n$ curves that are graphs of polynomial functions of constant maximum degree $s$ can be cut into an arrangement of $O\left(n^{2-1 / 3^{s-1}} \log ^{(3 / 2)\left(1-1 / 3^{s-1}\right)} n\right)$ extendible pseudo-segments.

Proof. Suppose we can cut any arrangement of any $n$ degree- $(s-1)$ polynomial functions into $O\left(n^{2-\alpha} \log ^{(3 / 2)(1-\beta)} n\right)$ extendible (1-intersecting) pseudo-segments. Apply this procedure to the derivatives of the given degree-s polynomial functions. Cut 
the graphs of the given functions at the corresponding $x$-values. Observation 6.1 tells us that the resulting arrangement is an arrangement of extendible pseudo-parabolic (2-intersecting) segments. Applying Theorem 5.1 with $N=O\left(n^{2-\alpha} \log ^{(3 / 2)(1-\beta)} n\right)$ and $X=O\left(n^{2}\right)$, we can further cut this arrangement into an arrangement of $O\left(n^{2-\alpha / 3} \log ^{(3 / 2)(1-\beta / 3)} n\right)$ extendible pseudo-segments. The theorem thus follows by induction (the base case $s=1$ is trivial).

Applying Theorem 2.1 to the output of Theorem 6.2 with

$$
N=O\left(n^{2-1 / 3^{s-1}} \log ^{(3 / 2)\left(1-1 / 3^{s-1}\right)} n\right) \text { and } X=O\left(n^{2}\right),
$$

we get a subquadratic $O\left(n^{2-2 / 3^{s}} \log ^{1-1 / 3^{s-1}} n\right)$ bound for the $k$-level. Again this bound can be refined by Agarwal et al.'s $k$-sensitizing techniques. Roughly the same bound holds for polynomial curve segments.

Corollary 6.3. The $k$-level in an arrangement of $n$ curves that are graphs of polynomial functions of a constant maximum degree s has $O\left(\lambda_{s}(n / k) k^{2-2 / 3^{s}} \log ^{1-1 / 3^{s-1}} k\right)$ vertices. For an arrangement of curve segments, the $k$-level has $O\left(\lambda_{s+2}(n / k) k^{2-2 / 3^{s}} \log ^{1-1 / 3^{s-1}} k\right)$ vertices.

Although polynomials are the most natural instances, the above proof clearly works for any curve families whose $(s-1)$ th derivatives obey the pseudo-line property.

\section{An Improvement}

Recently, Agarwal et al. [5] have improved Tamaki and Tokuyama's theorem [48] in various special cases; in particular, they have proved that every family of $n$ parabolas can be cut into $O\left(n^{3 / 2}(\log n)^{O\left(\alpha^{2}(n)\right)}\right)$ pseudo-segments. Their result immediately implies an improvement to our Theorem 6.2. The improvement though is small, because only the base case is affected in our proof (recall that our iterative step invokes a cut theorem for general pseudo-parabolas). In this section we describe a different approach that improves the iterative step.

The approach requires understanding how Tamaki and Tokuyama's proof works (readers are referred to their paper [48] for fuller details):

- A pair $(f, g)$ of curves (or curve segments) that intersect twice, with $f$ above $g$ at $x=-\infty$, is said to form a lens, denoted lens $(f, g)$ and defined as the boundary of the region bounded by $f$ and $g$; e.g., see the bold part of Fig. 3 (bottom). The first observation is that cutting pseudo-parabolas into pseudo-segments is basically the same as stabbing all lenses.

- A set of objects is nonoverlapping if each pair of objects intersects only at a discrete set of points. Using combinatorial hitting-set/set-cover bounds (with random sampling), Tamaki and Tokuyama showed that the minimal number of points to stab the lenses is basically of the same order as the maximal number of nonoverlapping lenses.

- This latter number is then bounded by elementary means: Given a nonoverlapping collection $L$ of lenses, we can view $L$ as a bipartite graph $\mathcal{G}(L)$, with the curves (or curve segments) as vertices, and pairs that form lenses in $L$ as edges. As it 


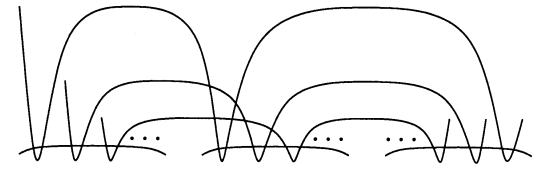

(a)

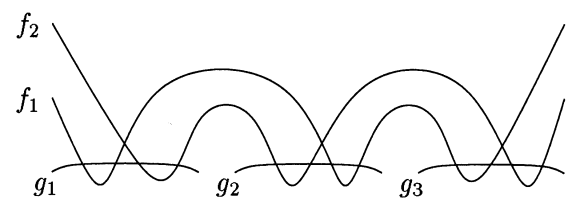

(b)

Fig. 4. (a) A $K_{c, 3}$ subgraph is possible for pseudo-parabolic segments. (b) Proof of Lemma 7.1: a $K_{c, 3}$ subgraph is impossible if $F$ is a pseudo-parabola family.

turns out, for pseudo-parabolas, $\mathcal{G}(L)$ cannot contain $K_{c, 3}$ as a subgraph for some constant $c$. It then follows easily (e.g., see [16]) that the number of edges in $\mathcal{G}(L)$ is $O\left(n^{5 / 3}\right)$.

Agarwal et al.'s improvement requires some more involved techniques to bound the maximal number of nonoverlapping lenses. Here, we follow Tamaki and Tokuyama's more elementary approach.

To warm up, we first consider the problem of generalizing Tamaki and Tokuyama's theorem to cut pseudo-parabolic segments. Notice that their proof does not work directly, as $K_{c, 3}$ can occur in $\mathcal{G}(L)$ for (nonextendible) pseudo-parabolic segments (see Fig. 4(a)). Furthermore, unlike pseudo-segments, there does not appear to be a natural way to make pseudo-parabolic segments extendible. Fortunately, the segment-tree idea from Section 3 still proves useful here and reduces the problem to a "bipartite" one: cutting lenses formed between pseudo-parabolas and pseudo-parabolic segments. This weaker problem can in fact be handled by Tamaki and Tokuyama's proof technique:

Lemma 7.1. Let $F$ be a family of $n$ pseudo-parabolas and let $G$ be a family of $n s$ intersecting curve segments for a fixed s, such that each member of $F$ intersects each member of $G$ at most twice. Then $F$ and $G$ can be cut into $O\left(n^{5 / 3}\right)$ subsegments such that each subsegment of $F$ intersects each subsegment of $G$ at most once.

Proof. By Tamaki and Tokuyama's technique, it suffices to demonstrate that if $L$ is a nonoverlapping collection of lenses formed by pairs in $F \times G$ (and, similarly, $G \times F$ ), then $\mathcal{G}(L)$ does not contain $K_{c, 3}$ (similarly, $K_{3, c}$ ) as a subgraph for some constant $c$. In other words, given $f_{1}, \ldots, f_{c} \in F$ and $g_{1}, g_{2}, g_{3} \in G$ such that each $\left(f_{i}, g_{j}\right)$ forms a lens and no two such lenses overlap, we have to derive a contradiction.

First observe that these lenses must all be formed with edges of the upper envelope $E$ of $\left\{g_{1}, g_{2}, g_{3}\right\}$, because if some $g_{k}$ lies above some point on lens $\left(f_{i}, g_{j}\right)$, then lens $\left(f_{i}, g_{k}\right)$ would overlap with lens $\left(f_{i}, g_{j}\right)$.

Since $E$ has at most a constant number of edges, for a sufficiently large $c$, we are guaranteed to find two curves, say $f_{1}$ and $f_{2}$, that form lenses with the same set of (three) edges of $E$. However, as Fig. 4(b) indicates, this scenario cannot happen, because $f_{1}$ and $f_{2}$ would intersect three times (at least) - once between (in terms of $x$-values) lens $\left(f_{1}, g_{j}\right)$ and lens $\left(f_{2}, g_{j}\right)$, for each $j \in\{1,2,3\}$.

Remark. The argument above for the $K_{3, c}$ exclusion is slightly different from Tamaki and Tokuyama's, and, in some ways, better. For example, our proof generalizes to an 
$O\left(n^{2-1 /(s+1)}\right)$ bound for cutting $s$-intersecting curves into $(s-1)$-intersecting curve segments for any even $s$ (by a $K_{s+1, c}$ exclusion); this improves Theorem 6.2 of [48].

Theorem 7.2. Every arrangement of $N$ pseudo-parabolic segments can be cut into an arrangement of $O\left(N^{5 / 3}\right)$ pseudo-segments. If $X$ is the number of intersecting pairs, then the bound improves to $O\left(N+N^{1 / 3} X^{2 / 3}\right)$.

Proof. We use divide-and-conquer in the style of the segment tree [43]. Suppose the $N$ segments are contained in a slab $\sigma$, with $h$ endpoints strictly inside $\sigma$. Divide the slab by a vertical line $\ell$ into two subslabs $\sigma_{1}$ and $\sigma_{2}$, each with $\leq h / 2$ endpoints strictly inside. Cut the segments at $\ell$. Take each subslab $\sigma_{i}(i \in\{1,2\})$. Classify a segment in $\sigma_{i}$ as short if at least one of its endpoints is strictly inside $\sigma_{i}$, long otherwise. Cut the $\leq h / 2$ short segments recursively. For the long segments, which behave like pseudo-parabolas, we can make $O\left(N^{5 / 3}\right)$ additional cuts, by Lemma 7.1, to ensure that each subsegment of the long segments intersects each subsegment of the long/short segments at most once. The total number of cuts satisfies the recurrence

$$
T(N, h)=2 T(h / 2, h / 2)+O\left(N^{5 / 3}\right),
$$

which solves to $T(N, h)=O\left(N^{5 / 3}\right)$. The intersection-sensitive bound now follows exactly as in the proof of Theorem 5.1.

Next, we consider the problem of cutting a family $S$ of 3-intersecting curve segments into 2-intersecting curve segments. Under the assumption that $S^{\prime}$ is pseudo-parabolic, we can get an $O\left(n^{5 / 3}\right)$ bound by cutting $S^{\prime}$ into pseudo-segments, as we have already observed in Section 6. Surprisingly, we can improve this bound to $O\left(n^{3 / 2}\right)$ under the same assumption, by directly modifying the proofs of Lemma 7.1 and Theorem 7.2 to exploit the relationship between the $S^{\prime}$ and $S$ families. As it turns out, in this setting, we can actually show a $K_{2, c}$ exclusion!

In the subsequent proof, a pair $(f, g)$ of curves (or curve segments) that intersect three times, with $f$ below $g$ at $x=-\infty$, is said to form a double-lens, double-lens $(f, g)$, defined as the boundary of the two regions bounded by $f$ and $g$; e.g., see the bold part of Fig. 3 (top).

Lemma 7.3. Suppose $F^{\prime}$ forms a family of $n$ pseudo-parabolas and $G^{\prime}$ forms a family of $n$ s-intersecting curve segments for a fixed s, such that each member of $F^{\prime}$ intersects each member of $G^{\prime}$ at most twice. Then $F$ and $G$ can be cut into $O\left(n^{3 / 2}\right)$ subsegments such that each subsegment of $F$ intersects each subsegment of $G$ at most twice.

Proof. By Observation 6.1, each member of $F$ intersects each member of $G$ at most three times, so the goal is to stab all double-lenses formed by pairs in $(F \times G) \cup(G \times F)$. By Tamaki and Tokuyama's technique, it suffices to demonstrate that if $L$ is a nonoverlapping collection of double-lenses formed by pairs in $F \times G$ (resp. $G \times F$ ), then $\mathcal{G}(L)$ does 


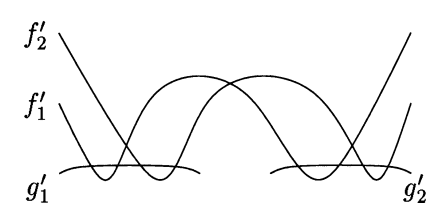

(a)

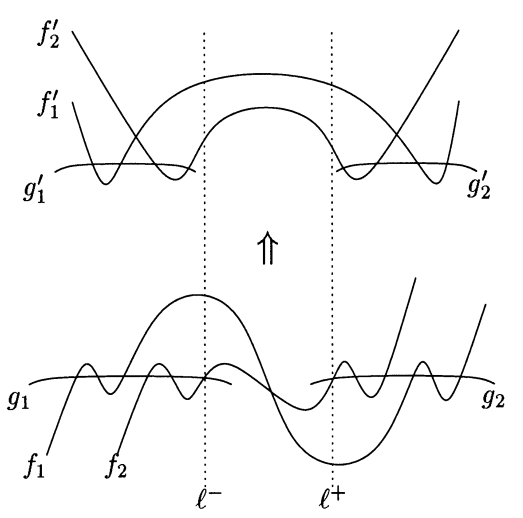

(b)

Fig. 5. Proof of Lemma 7.3: (a) Case 1 is impossible; (b) Case 2 is impossible (somewhere between $\ell^{-}$and $\ell^{+}, f_{1}$ has a smaller slope than $f_{2}$ ).

not contain $K_{c, 2}$ (resp. $K_{2, c}$ ) as a subgraph for some constant $c$. In other words, given $f_{1}, \ldots, f_{c} \in F$ and $g_{1}, g_{2} \in G$ such that each $\left(f_{i}, g_{j}\right)$ forms a double-lens and no two such double-lenses overlap, we have to derive a contradiction.

Observe that if $(f, g)$ forms a double-lens, then $\left(f^{\prime}, g^{\prime}\right)$ forms a lens with lens $\left(f^{\prime}, g^{\prime}\right)$ $\subseteq$ [double-lens $(f, g)]^{\prime}$ (see Fig. 3). Each $\left(f_{i}^{\prime}, g_{j}^{\prime}\right)$ thus forms a lens and no two such lenses overlap. It makes sense to apply arguments from our earlier proof of Lemma 7.1 to analyze the derivative families $F^{\prime}$ and $G^{\prime}$. In particular, for a sufficiently large $c$, we can infer the existence of two curves, say $f_{1}^{\prime}$ and $f_{2}^{\prime}$, that form lenses with the same set of (two) edges of the upper envelope of $\left\{g_{1}^{\prime}, g_{2}^{\prime}\right\}$. Up to symmetry, the lenses formed by $f_{1}^{\prime}$ and $f_{2}^{\prime}$ can be arranged in only two different ways, from left to right:

- Case 1: lens $\left(f_{1}^{\prime}, g_{1}^{\prime}\right)$, lens $\left(f_{2}^{\prime}, g_{1}^{\prime}\right)$, lens $\left(f_{1}^{\prime}, g_{2}^{\prime}\right)$, lens $\left(f_{2}^{\prime}, g_{2}^{\prime}\right)$.

As Fig. 5(a) indicates, this case cannot happen, because $f_{1}^{\prime}$ and $f_{2}^{\prime}$ would intersect three times (at least) - between lens $\left(f_{1}^{\prime}, g_{1}^{\prime}\right)$ and lens $\left(f_{2}^{\prime}, g_{1}^{\prime}\right)$, between lens $\left(f_{2}^{\prime}, g_{1}^{\prime}\right)$ and lens $\left(f_{1}^{\prime}, g_{2}^{\prime}\right)$, and between lens $\left(f_{1}^{\prime}, g_{2}^{\prime}\right)$ and lens $\left(f_{2}^{\prime}, g_{2}^{\prime}\right)$.

- Case 2: lens $\left(f_{1}^{\prime}, g_{1}^{\prime}\right)$, lens $\left(f_{2}^{\prime}, g_{1}^{\prime}\right)$, lens $\left(f_{2}^{\prime}, g_{2}^{\prime}\right)$, lens $\left(f_{1}^{\prime}, g_{2}^{\prime}\right)$.

As Fig. 5(b) (top) indicates, $f_{1}^{\prime}$ must be above $f_{2}^{\prime}$ between $\operatorname{lens}\left(f_{2}^{\prime}, g_{1}^{\prime}\right)$ and lens $\left(f_{2}^{\prime}, g_{2}^{\prime}\right)$.

This case also cannot happen, but to see this, we need to return to the original families $F$ and $G$. We know that double-lens $\left(f_{1}, g_{1}\right)$ is left of double-lens $\left(f_{2}, g_{1}\right)$, which is left of double-lens $\left(f_{2}, g_{2}\right)$, which is left of double-lens $\left(f_{1}, g_{2}\right)$, as shown in Fig. 5(b) (bottom). Now, in order to avoid a fourth intersection between $f_{1}$ and $g_{1}, f_{1}$ must be above $f_{2}$ immediately to the right of double-lens $\left(f_{2}, g_{1}\right)$; on the other hand, to avoid a fourth intersection between $f_{1}$ and $g_{2}, f_{1}$ must be below $f_{2}$ immediately to the left of double-lens $\left(f_{2}, g_{2}\right)$. So, in between double-lens $\left(f_{2}, g_{1}\right)$ and double-lens $\left(f_{2}, g_{2}\right), f_{1}$ and $f_{2}$ must intersect, and at the first intersection, $f_{1}^{\prime}$ would be smaller than $f_{2}^{\prime}$. However, this contradicts the previous paragraph. 
Theorem 7.4. If $S^{\prime}$ forms a family of $N$ pseudo-parabolic segments, then $S$ can be cut into $O\left(N^{3 / 2}\right)$ pseudo-parabolic segments. If $X$ is the number of intersecting pairs, then the bound improves to $O\left(N+N^{1 / 2} X^{1 / 2}\right)$.

Proof. By Lemma 7.3, as in the proof of Theorem 7.2. The intersection-sensitive bound also follows as in the proof of Theorem 5.1.

With Theorem 7.4, an improvement to Theorem 6.2 now follows simply:

Theorem 7.5. Any arrangement of curves that are graphs of $n$ polynomials of maximum degree $s \geq 2$ can be cut into an arrangement of $O\left(n^{2-1 / 2^{s-2}}\right)$ pseudo-parabolic segments, or $O\left(n^{2-1 /\left(3 \cdot 2^{s-2}\right)}\right)$ pseudo-segments, or $O\left(n^{2-1 /\left(3 \cdot 2^{s-2}\right)} \log n\right)$ extendible pseudo-segments.

Proof. The first part can be proved by induction: suppose we can cut the arrangement of any $n$ degree- $(s-1)$ polynomials into $O\left(n^{2-\alpha}\right)$ pseudo-parabolic segments. Then given $n$ degree-s polynomials $S$, we can first cut $S^{\prime}$ into $O\left(n^{2-\alpha}\right)$ pseudo-parabolic segments and then apply Theorem 7.4 (with $N=O\left(n^{2-\alpha}\right)$ and $X=O\left(n^{2}\right)$ ) to cut $S$ into $O\left(n^{2-\alpha / 2}\right)$ pseudo-parabolic segments.

The second part follows by applying Theorem 7.2 (with $N=O\left(n^{2-1 / 2^{s-2}}\right)$ and $X=O\left(n^{2}\right)$ ) to cut the resulting pseudo-parabolic segments into pseudo-segments. The last part follows by applying Theorem 3.3 to cut these pseudo-segments into extendible pseudo-segments.

Now, applying Theorem 2.1 with $N=O\left(n^{2-1 /\left(3 \cdot 2^{s-2}\right)} \log n\right)$ and $X=O\left(n^{2}\right)$ yields the improvement to Corollary 6.3:

Corollary 7.6. The $k$-level in an arrangement of $n$ curves that are graphs of polynomial functions of a constant maximum degree s has $O\left(\lambda_{s}(n / k) k^{2-1 /\left(9 \cdot 2^{s-3}\right)} \log ^{2 / 3} k\right)$ vertices. For an arrangement of curve segments, the $k$-level has $O\left(\lambda_{s+2}(n / k) k^{2-1 /\left(9 \cdot 2^{s-3}\right)} \log ^{2 / 3} k\right)$ vertices.

\section{Other Applications}

Given an arrangement of curves (or curve segments), a chain is a connected subset of the union that intersects each vertical line at most once. A point $v$ is a vertex of the chain if, around $v$, two different curves (curve segments) appear on the chain; say the one appearing to the left of $v$ is $s_{1}$ and the one appearing to the right of $v$ is $s_{2}$. If immediately to the left of $v, s_{1}$ is below $s_{2}$, then $v$ is said to be a pseudo-concave vertex of the chain. If all of its vertices are pseudo-concave, then the chain is pseudoconcave.

Agarwal et al. [1] observed that the complexity of the $k$-level can be bounded by the complexity of a nonoverlapping collection of (pseudo-)concave chains. In fact, Dey's breakthrough [21] was in part inspired by this point of view; his proof yields a tight 
$O\left(n k^{1 / 3}+n^{2 / 3} k^{2 / 3}\right)$ bound for $k$ nonoverlapping concave chains in an arrangement of lines (or pseudo-lines, with the lemma by Tamaki and Tokuyama [47]). We will show that our level bounds can be carried over to this more general problem as well for the curve families we have considered. First, we need to make Dey's bound intersectionsensitive with the following analogue of Theorem 2.1. This time, the proof requires the random-sampling approach. In the following, a chain is proper if its leftmost point is an endpoint.

Theorem 8.1. A nonoverlapping collection of proper pseudo-concave chains in an arrangement of $N$ extendible pseudo-segments with $X$ intersecting pairs has $O(N+$ $\left.N^{2 / 3} X^{1 / 3}\right)$ vertices.

Proof. Take a random sample $R$ of $r$ segments. Inside each trapezoid $\Delta \in \operatorname{VD}(R)$, we have a nonoverlapping collection of proper pseudo-concave chains in the arrangement of $S_{\Delta}$ clipped to $\Delta$. This collection can also be viewed as $O\left(\left|S_{\Delta}\right|\right)$ pseudo-concave chains in the arrangement of the extensions of $S_{\Delta}$, and, by Dey's bound, has $O\left(\left|S_{\Delta}\right|^{4 / 3}\right)$ vertices. The total number of vertices in the chains is therefore $O(M)$, where

$$
M=\sum_{\Delta \in \mathrm{VD}(R)}\left|S_{\Delta}\right|^{4 / 3}
$$

By Clarkson and Shor's analysis [20], [40],

$$
E[M]=O\left(\left(r+X(r / N)^{2}\right) \cdot(N / r)^{4 / 3}\right) .
$$

The proof is completed by setting $r=\left\lceil N^{2} / X\right\rceil$ (if $X \geq N$ ).

When we cut an arrangement, the cut chains remain nonoverlapping and pseudoconcave. To make chains proper, we can cut the arrangement at each of their $k$ leftmost points (extendibility is maintained). So, for a pseudo-parabola arrangement, we can apply Tamaki and Tokuyama's theorem with Theorem 3.3, substituting $N=O\left(n^{5 / 3} \log n+k\right)$ and $X=O\left(n^{2}\right)$ in Theorem 8.1. For an arrangement of polynomial curves, we can apply Theorem 7.5, substituting $N=O\left(n^{2-1 /\left(3 \cdot 2^{s-2}\right)} \log n+k\right)$ and $X=O\left(n^{2}\right)$.

Corollary 8.2. A nonoverlapping collection of $k$ pseudo-concave chains in an arrangement of $n$ pseudo-parabolas has $O\left(n^{16 / 9} \log ^{2 / 3} n+n^{2 / 3} k^{2 / 3}\right)$ vertices.

Corollary 8.3. A nonoverlapping collection of $k$ pseudo-concave chains in an arrangement of $n$ curves that are graphs of polynomial functions of a constant maximum degree s has $O\left(n^{2-1 /\left(9 \cdot 2^{s-3}\right)} \log ^{2 / 3} n+n^{2 / 3} k^{2 / 3}\right)$ vertices.

Previously, Tamaki and Tokuyama [48] gave a weaker $O\left(n^{11 / 6}\right)$ bound for a single pseudo-concave chain $(k=1)$ in a pseudo-parabola arrangement. For multiple chains, they had a complicated proof of a bound of $O\left(n^{43 / 22}+n^{23 / 12} k^{1 / 12}\right)$, which is subquadratic only when $k=o(n)$. For a single pseudo-concave chain, they observed a lower bound of $\Omega\left(n^{4 / 3}\right)$ for parabolas, and, interestingly, a lower bound of $\Omega\left(n^{2} / \log n\right)$ for 3-intersecting 


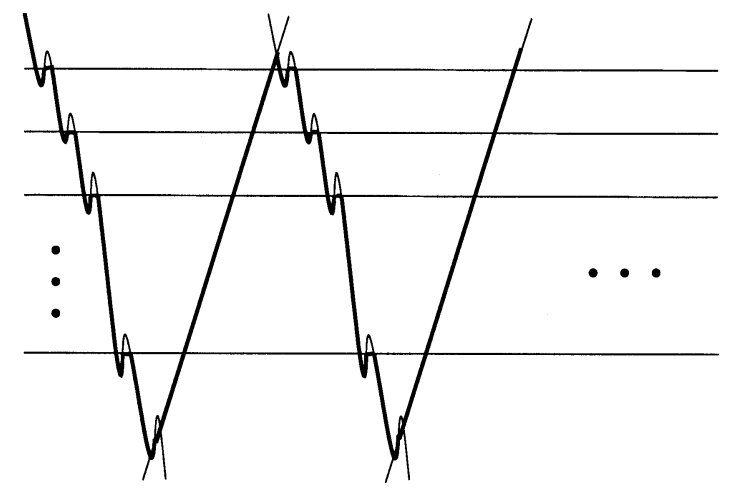

Fig. 6. A pseudo-concave chain of quadratic size in an arrangement of 3-intersecting curves.

curves (actually, see Fig. 6 for an $\Omega\left(n^{2}\right)$ example); hence, no generalization of Corollary 8.3 to arbitrary $s$-intersecting curves is possible!

Standard reductions [26], [32], [36] reveal a connection of the multiple pseudoconcave chain problem to an important graph problem, studied by Gusfield and others: bounding the number of changes to the minimum spanning tree in a graph with parametric edge weights, or, more generally, the minimum-weight basis in a parametric matroid. For the case of linearly varying weights, Dey's bound [21] gives $O\left(m n^{1 / 3}\right)$ (which matches a lower bound by Eppstein [26] for the matroid generalization of the problem); here, $m$ is the number of edges and $n$ is the number of vertices. For the case of polynomially varying weights, we immediately have the first nontrivial bound from Corollary 8.3:

Corollary 8.4. A parametric minimum spanning tree with $m$ edges changes at most $O\left(m^{2-1 /\left(9 \cdot 2^{s-3}\right)} \log ^{2 / 3} m\right)$ times, if the edge weights are polynomial functions of constant maximum degree $s$ in time.

The geometric version of the above problem, i.e., minimum spanning trees of moving points in a fixed-dimensional space, has also generated much interest. Applying Corollary 8.4 with observations made by Katoh et al. [36], we get the first subcubic result:

Corollary 8.5. The $L_{p}$-minimum spanning tree of $n$ moving points in $\mathbb{R}^{d}$ changes at most $O\left(\lambda_{p s+2}(n) n^{2-1 /\left(9 \cdot 2^{p s-3}\right)} \log ^{2 / 3} n\right)$ times, if $p$ and $d$ are constant positive integers and the coordinates of the points are polynomial functions of constant maximum degree s in time.

The most noteworthy case of the above is perhaps the Euclidean minimum spanning tree of linearly moving points ( $p=2, s=1$ ), where Corollary 8.5 gives a bound of $O\left(n^{25 / 9} 2^{\alpha(n)} \log ^{2 / 3} n\right)$-the first improvement over Katoh et al.'s $O\left(n^{3} 2^{\alpha(n)}\right)$ bound [36]. (Tamaki and Tokuyama [48] were unable to obtain such an improvement because their result on multiple chains for pseudo-parabolas was too weak.) By Agarwal et al.'s re- 
cently improved cut theorem for parabolas [5], the bound can be further reduced to $O\left(n^{8 / 3}(\log n)^{O\left(\alpha^{2}(n)\right)}\right)$.

For algorithms on the parametric/kinetic minimum spanning tree problem in both its graph and geometric settings, see [3] and [13].

Very recently, our Theorem 3.3 was used by Agarwal et al. [2] to bound the combinatorial complexity of multiple faces in arrangements of pseudo-segments and of circles. Our Theorem 6.2/7.5 was also used by Aronov and Sharir [10] to derive improved bounds on the number of incidences between points and graphs of polynomials.

\section{Open Problems}

We close with some interesting questions.

1. Can we improve our $O(n \log n)$ bound for cutting pseudo-segments into extendible pseudo-segments, or is there a superlinear lower bound?

2. Can we improve Tamaki and Tokuyama's $O\left(n^{5 / 3}\right)$ bound for cutting general pseudo-parabolas into pseudo-segments? Tamaki and Tokuyama observed an $\Omega\left(n^{4 / 3}\right)$ lower bound [48] which holds for parabolas.

3. Can we improve the $O\left(n^{3 / 2}\right)$ bound in Theorem 7.4? If so, further improvements to our results for polynomials would follow. We know of no nontrivial lower bound here. Can we prove an $o\left(n^{3 / 2}\right)$ bound for cutting graphs of degree-s polynomials into $(s-1)$-intersecting curve segments for some $s \geq 3$ ?

4. What is the best bound for cutting graphs of degree- $s$ polynomials into pseudosegments for a given fixed $s$ ? Does the exponent have to converge to 2 exponentially, as in our current result? Does it have to depend on $s$ at all?

5. Finally, can we obtain nontrivial bounds on the $k$-level for a more general class of curves beyond graphs of polynomials? Fixed-degree algebraic curves are of particular interest. Our proof works only if after taking a constant number of derivatives, we get a pseudo-line or pseudo-parabolic arrangement; for instance, we do not see how to generalize our result to rational functions or parametrically defined polynomial curves. With the failure of the cutting approach, the question for arbitrary $s$-intersecting curve families remains as perplexing as before. Can we prove a lower bound better than Toth's [49] for curves?

\section{References}

1. P. K. Agarwal, B. Aronov, T. M. Chan, and M. Sharir. On levels in arrangements of lines, segments, planes, and triangles. Discrete Comput. Geom., 19:315-331, 1998.

2. P. K. Agarwal, B. Aronov, and M. Sharir. On the complexity of many faces in arrangements of circles. In Proc. 42nd IEEE Sympos. Found. Comput. Sci., pages 74-83, 2001.

3. P. K. Agarwal, D. Eppstein, L. J. Guibas, and M. R. Henzinger. Parametric and kinetic minimum spanning trees. In Proc. 39th IEEE Sympos. Found. Comput. Sci., pages 596-605, 1998.

4. P. K. Agarwal and J. Erickson. Geometric range searching and its relatives. In Advances in Discrete and Computational Geometry (B. Chazelle, J. E. Goodman, and R. Pollack, eds.), AMS Press, Providence, RI, pages 1-56, 1999. 
5. P. K. Agarwal, E. Nevo, J. Pach, R. Pinchasi, M. Sharir, and S. Smorodinsky. Lenses in arrangements of pseudo-circles and their applications. Manuscript, 2001.

6. P. K. Agarwal and M. Sharir. Arrangements and their applications. In Handbook of Computational Geometry (J. Urrutia and J. Sack, eds.), North-Holland, Amsterdam, pages 49-119, 2000.

7. N. Alon and E. Győri. The number of small semispaces of a finite set of points in the plane. J. Combin. Theory Ser. A, 41:154-157, 1986.

8. A. Andrzejak and E. Welzl. $k$-Sets and $j$-facets: a tour of discrete geometry. Manuscript, 1997.

9. B. Aronov, B. Chazelle, H. Edelsbrunner, L. J. Guibas, M. Sharir, and R. Wenger. Points and triangles in the plane and halving planes in space. Discrete Comput. Geom., 6:435-442, 1991.

10. B. Aronov and M. Sharir. Cutting circles into pseudo-segments and improved bounds for incidences. Discrete Comput. Geom., 28:475-490, 2002.

11. M. Atallah. Some dynamic computational geometry problems. Comput. Math. Appl., 11:1171-1181, 1985.

12. I. Bárány, Z. Füredi, and L. Lovász. On the number of halving planes. Combinatorica, 10:175-183, 1990.

13. J. Basch, L. Guibas, and L. Zhang. Proximity problems on moving points. In Proc. 13th ACM Sympos. Comput. Geom., pages 344-351, 1997.

14. M. de Berg and O. Schwarzkopf. Cuttings and applications. Internat. J. Comput. Geom. Appl., 5:343-355, 1995.

15. S. Bespamyatnikh, D. Kirkpatrick, and J. Snoeyink. Generalizing ham sandwich cuts to equitable subdivisions. Discrete Comput. Geom., 24:605-622, 2000.

16. B. Bollobás. Modern Graph Theory. Graduate Texts in Mathematics, 184. Springer-Verlag, New York, 1998.

17. T. M. Chan. Remarks on $k$-level algorithms in the plane. Manuscript, 1999.

18. T. M. Chan. On levels in arrangements of curves. In Proc. 41 st IEEE Sympos. Found. Comput. Sci., pages 219-227, 2000.

19. B. Chazelle and F. P. Preparata. Halfspace range search: an algorithmic application of $k$-sets. Discrete Comput. Geom., 1:3-93, 1986.

20. K. L. Clarkson and P. W. Shor. Applications of random sampling in computational geometry, II. Discrete Comput. Geom., 4:387-421, 1989.

21. T. K. Dey. Improved bounds on planar $k$-sets and $k$-levels. Discrete Comput. Geom., 19:373-382, 1998.

22. T. K. Dey and H. Edelsbrunner. Counting triangle crossings and halving planes. Discrete Comput. Geom., 12:281-289, 1994

23. H. Edelsbrunner. Algorithms in Combinatorial Geometry. Springer-Verlag, Berlin, 1987.

24. H. Edelsbrunner, L. J. Guibas, J. Pach, R. Pollack, R. Seidel, and M. Sharir. Arrangements of curves in the plane: topology, combinatorics, and algorithms. Theoret. Comput. Sci., 92:319-336, 1992.

25. D. Eppstein. Improved bounds for intersecting triangles and halving planes. J. Combin. Theory Ser. A, 62:176-182, 1993.

26. D. Eppstein. Geometric lower bounds for parametric matroid optimization. Discrete Comput. Geom., 20:463-476, 1998.

27. P. Erdős, L. Lovász, A. Simmons, and E. Straus. Dissection graphs of planar point sets. In A Survey of Combinatorial Theory (J. N. Srivastava, ed.), North-Holland, Amsterdam, pages 139-154, 1973.

28. H. Everett, J.-M. Robert, and M. van Kreveld. An optimal algorithm for the $(\leq k)$-levels, with applications to separation and transversal problems. Internat. J. Comput. Geom. Appl., 6:247-261, 1996.

29. J. E. Goodman and R. Pollack. On the number of $k$-subsets of a set of $n$ points in the plane. J. Combin. Theory Ser. A, 36:101-104, 1984.

30. J. E. Goodman and R. Pollack. Allowable sequences and order types in discrete and computational geometry. In New Trends in Discrete and Computational Geometry, Algorithms and Combinatorics, vol. 10 (J. Pach, ed.), Springer-Verlag, New York, pages 103-134, 1993.

31. L. J. Guibas. Kinetic data structures: a state of the art report. In Proc. $3 r d$ Workshop Algorithmic Found. Robotics, pages 191-209, 1998.

32. D. Gusfield. Bounds for the parametric minimum spanning tree problem. In Proc. Humboldt Conf. Graph Theory, Combin. Comput., Utilitas Mathematica, Winnipeg, Manitoba, pages 173-183, 1979.

33. D. Halperin. Arrangements. In Handbook of Discrete and Computational Geometry (J. E. Goodman and J. O'Rourke, eds.), CRC Press, Boca Raton, FL, pages 389-412, 1997.

34. S. Har-Peled. Taking a walk in a planar arrangement. In Proc. 40th IEEE Sympos. Found. Comput. Sci., pages 100-110, 1999. 
35. N. Katoh and T. Tokuyama. Lovász's lemma for the three-dimensional $k$-level of concave surfaces and its applications. In Proc. 40th IEEE Sympos. Found. Comput. Sci., pages 389-398, 1999.

36. N. Katoh, T. Tokuyama, and K. Iwano. On minimum and maximum spanning trees of linearly moving points. Discrete Comput. Geom., 13:161-176, 1995.

37. L. Lovász. On the number of halving lines. Ann. Univ. Sci. Budapest. Eötvös Sect. Math., 14:107-108, 1971.

38. C.-Y. Lo, J. Matoušek, and W. L. Steiger. Algorithms for ham-sandwich cuts. Discrete Comput. Geom., 11:433-452, 1994.

39. J. Matoušek. On geometric optimization with few violated constraints. Discrete Comput. Geom., 14:365384, 1995.

40. K. Mulmuley. Computational Geometry: An Introduction Through Randomized Algorithms. Prentice-Hall, Englewood Cliffs, NJ, 1994.

41. J. Pach and P. K. Agarwal. Combinatorial Geometry. Wiley-Interscience, New York, 1995.

42. J. Pach, W. Steiger, and E. Szemerédi. An upper bound on the number of planar $k$-sets. Discrete Comput. Geom., 7:109-123, 1992.

43. F. P. Preparata and M. I. Shamos. Computational Geometry: An Introduction. Springer-Verlag, New York, 1985.

44. M. Sharir. On $k$-sets in arrangements of curves and surfaces. Discrete Comput. Geom., 6:593-613, 1991.

45. M. Sharir and P. K. Agarwal. Davenport-Schinzel Sequences and Their Geometric Applications. Cambridge University Press, New York, 1995.

46. M. Sharir, S. Smorodinsky, and G. Tardos. An improved bound for $k$-sets in three dimensions. In Proc. 16th ACM Sympos. Comput. Geom., pages 43-49, 2000.

47. H. Tamaki and T. Tokuyama. A characterization of planar graphs by pseudo-line arrangements. In Proc. 8th Internat. Sympos. Algorithms and Computation, Lecture Notes in Computer Science, vol. 1350, SpringerVerlag, Berlin, pages 123-132, 1997.

48. H. Tamaki and T. Tokuyama. How to cut pseudo-parabolas into segments. Discrete Comput. Geom., 19:265-290, 1998.

49. G. Toth. Point sets with many $k$-sets. In Proc. 16th ACM Sympos. Comput. Geom., pages 37-42, 2000.

50. R. Živaljević and S. Vrećica. The colored Tverberg's problem and complexes of injective functions. J. Combin. Theory Ser. A, 61:309-318, 1992.

Received January 29, 2002, and in revised form March 21, 2002. Online publication January 30, 2003. 